\title{
PREVISÃO DA REQUEIMA DA BATATEIRA
}

\section{RODRIGO V. COSTA, LAÉRCIO ZAMBOLIM, FRANCISCO X. RIBEIRO DO VALE \& EDUARDO S. G. MIZUBUTI}

\author{
Departamento de Fitopatologia, Universidade Federal de Viçosa, CEP 36.571-000, Viçosa-MG, \\ Fone (31) 3899-1095, e-mail: zambolim@ufv.br
}

(Aceito para publicação em 12/04/2002)

Autor para correspondência: Laércio Zambolim

COSTA, R.V., ZAMBOLIM, L., VALE, F.X.R. \& MIZUBUTI, E.S.G. Previsão da requeima da batateira. Fitopatologia Brasileira 27:349354. 2002.

\section{RESUMO}

Com o objetivo de adequar um sistema de previsão para uso criterioso do controle químico da requeima da batateira (Solanum tuberosum), foram instalados ensaios em condição de campo, seguindo-se o delineamento experimental em blocos ao acaso, com sete tratamentos e quatro repetições, com a cultivar Bintje. Os fungicidas dimetomorfe, cimoxanil, mancozeb e clorotalonil foram associados com diferentes esquemas de pulverização baseados no sistema de previsão modificado de Wallin (1962) e no sistema tradicional de controle da requeima. Os tratamentos consistiram de : 1) aplicação de dimetomorfe de acordo com VSD-12 + clorotalonil semanalmente; 2) aplicação de dimetomorfe de acordo com VSD-14 + clorotalonil semanalmente; 3) aplicação de dimetomorfe de acordo com VSD-16 + clorotalonil semanalmente; 4) aplicação dos fungicidas protetores mancozeb, clorotalonil e oxicloreto de cobre alternados a cada sete dias; 5) aplicação dos fungicidas mancozeb, dimetomorfe, clorotalonil e cimoxanil, alternados a cada sete dias; 6) aplicação de dimetomorfe + clorotalonil de acordo com VSD-12; e 7) testemunha (sem aplicação). $\mathrm{O}$ uso do sistema de previsão conforme os tratamentos 1,2 e 3 apresentou eficiência comparável ao calendário semanal (tratamento 5) de pulverização no controle da requeima da batateira. Os tratamentos baseados no acúmulo de 12 e 14 VSD's apresentaram maior eficiência em prever a ocorrência de epidemias da requeima. Apenas o sistema de previsão 2, permitiu reduzir o número de pulverizações com fungicida sistêmico, sem que houvesse perda significativa na produção.

Palavras-chave adicionais: Solanum tuberosum, fungicida, sistema de aviso.

\section{ABSTRACT \\ Forecast system of potato late blight}

This experiment was carried out on a potato (Solanum tuberosum) crop to evaluate a prediction system to control late blight caused by Phytophthora infestans. The trial was conducted from May to September 1998 using the cultivar Bintje. Dimethomorph, cymoxanil, mancozeb and chorothalonil were associated with different spraying schemes, based on the modified Wallin (1962) prediction system and on the traditional late blight control system. The prediction schemes tested consisted of accumulated disease severity values (DSV) of 12, 14, and 16 . The traditional late blight control consisted of weekly applications of fungicides starting two weeks after plant emergence, while those defined by the prediction system were carried out based on the DSV accumulated throughout the experiment. Descriptive and diagrammatic scales were used to assess late blight severity. The efficiency of the spray schemes was compared with the area below the curve of disease progress and tuber yield. The treatments based on the prediction scheme were as efficient as the weekly calendar program in reducing disease intensity. Disease severity values of 12 and 14 allowed reduction in the number of sprays without significantly affecting the control level of the disease and tuber yield. The use of the systemic fungicide alone, based on the prediction scheme, resulted in high disease intensity; the use of protective fungicides at weekly intervals gave intermediate control similar to the weekly spray calendar. The prediction schemes based on DSV of 14 reduced dimethomorph sprays without any yield loss.

\section{INTRODUÇÃO}

Vários são os fatores que limitam a produção de batata (Solanum tuberosum L.) no Brasil, e dentre estes, as doenças têm ocupado lugar de destaque. A requeima causada pelo fungo Phytophthora infestans (Mont.) De Bary é uma das mais importantes, podendo comprometer todo o campo de produção em questão de poucos dias (Stevenson, 1983; Lopes \& Santos, 1994).

A presença da requeima da batateira no Brasil é anterior a 1913 (Lorena, 1913) e ocorre em praticamente todos os locais onde essa olerícola é cultivada, sendo mais severa sob alta umidade relativa do ar e temperaturas moderadas, 12 - $20{ }^{\circ} \mathrm{C}$. Em temperaturas acima de $30^{\circ} \mathrm{C}$ a requeima dificilmente ocorre, mas o patógeno permanece vivo, podendo provocar danos assim que as condições climáticas se tornarem favoráveis (Lopes \& Santos, 1994; Zambolim et al., 2000).

A cultura da batata tem sido cultivada em épocas favoráveis ao desenvolvimento da doença. O problema tornase ainda maior com o uso de cultivares suscetíveis, como 
Bintje. Os produtores insistem no plantio dessa cultivar devido à sua alta produtividade e boas características agronômicas certos de que poderão controlar a requeima com o uso de produtos químicos em caráter preventivo.

Segundo Nazareno et al. (1999), produtores da cultivar Bintje temerosos dos riscos de perdas inerentes à doença, iniciam as pulverizações com fungicidas preventivos tão logo inicia a expansão das primeiras folhas e seguem aplicando até o final do ciclo da planta, com intervalos de três a cinco dias. Quando as condições climáticas favorecem a doença, as aplicações são diárias. De acordo com Vale et al. (1992), as pulverizações são feitas sem a observação de critério de ocorrência da doença e, ou, de condições ambientais favoráveis.

Vários são os problemas decorrentes do uso excessivo de produtos químicos para o controle da requeima, como: aumento do custo de produção, intoxicação de aplicadores, contaminação do meio ambiente pela deriva e lixiviação dos excessos de produtos pulverizados, resíduos de fungicidas em tubérculos e outros órgãos vegetais (Zambolim et al., 1990) e surgimento de isolados do patógeno resistentes a fungicidas (Royle \& Shaw, 1988). Todas essas implicações têm sido motivo de grande preocupação, e têm levado à procura crescente por práticas e estratégias de controle da requeima que sejam eficientes, racionais e economicamente viáveis. Dentre essas alternativas, destacam-se as práticas e estratégias de manejo que objetivam integrar informações da biologia do patógeno e suas interações com as variáveis climáticas. De acordo com Chalfoun \& Lima (1986), a umidade relativa e a temperatura do ar influenciam a incidência e o desenvolvimento das doenças de forma direta e indireta. De forma direta, afetam o desenvolvimento do patógeno nas suas diversas fases do ciclo de vida. Indiretamente, afetam a suscetibilidade das plantas.

O emprego de sistemas de previsão tem se destacado como uma alternativa para otimizar o uso de produtos químicos, uma vez que auxiliam o processo de tomada de decisão, indicando períodos de condições favoráveis ao desenvolvimento das doenças, e determinando o momento adequado para as aplicações de fungicidas (Hyre, 1954; Pitblado, 1988). Dentre as vantagens do uso de sistemas de previsão pode-se citar: maior lucro ao produtor, decréscimo do risco de ocorrência de epidemias, redução do número de pulverizações e menor dano ao homem e ao meio ambiente (Bergamin Filho \& Amorim, 1995).

Os calendários de pulverização adotados pelos produtores são, na maioria das vezes, excessivos e não discriminatórios, desconsiderando a interação entre aspectos biológicos do ciclo de vida do patógeno e as condições climáticas. Além disso, apenas eventualmente apresentam controle efetivo e racional da doença. Baseado nessas evidências, os objetivos deste trabalho foram: avaliar a viabilidade de uso do modelo de previsão modificado de Wallin (1962) no controle da requeima; definir o intervalo de aplicação de fungicidas baseado no referido modelo, e avaliar a eficiência de fungicidas sistêmicos e protetores em culturas manejadas com sistema de previsão e no modo tradicional.

\section{MATERIAL E MÉTODOS}

\section{Condução do experimento}

Foram conduzidos no campo de pesquisas do Departamento de Fitotecnia da Universidade Federal de Viçosa, experimentos no período de maio a setembro de 1998 , empregando-se a cultivar Bintje, altamente suscetível à requeima. A semeadura foi realizada no espaçamento de 0,5 $\mathrm{m}$ entre plantas e $1 \mathrm{~m}$ entre fileiras, com tubérculos de $60 \pm$ $10 \mathrm{~g}$, com brotações uniformes.

Foi utilizado o delineamento experimental em blocos ao acaso, com sete tratamentos e quatro repetições, sendo cada parcela constituída de 24 plantas, dispostas em duas fileiras. Entre cada parcela foi deixada uma fileira de plantas como bordadura, com o objetivo de minimizar as interferências entre parcelas.

Os tratamentos foram constituídos da aplicação de fungicidas sistêmicos e protetores de acordo com o sistema de previsão e com o sistema convencional de controle da requeima (Tabela 1). Foram utilizados os fungicidas sistêmicos dimetomorfe (Forum 500 PM - 0,4 g/l) e cimoxanil (Curzate M 80 PM + Zinco PM - 1,5 g/l), e os protetores clorotalonil (Daconil 500 PM - 2 g/l), mancozeb (Manzate 800 PM - 3 g/l) e oxicloreto de cobre (Cobox 350 PM - 4 g/l). As pulverizações foram realizadas com pulverizador costal manual de 20 litros de capacidade, ao qual foi acoplado um bico de pulverização do tipo cone vazio.

\section{Obtenção das variáveis microclimáticas}

As variáveis microclimáticas (temperatura, umidade relativa e molhamento foliar) foram obtidas através de um termohigrohumectógrafo (G. Lufft GMbH8 Co.). O equipamento foi acondicionado em abrigo de madeira $(82 \times 72 \times 80$ $\mathrm{cm}$ ) suspenso $1,5 \mathrm{~m}$ do solo, entre as plantas. $\mathrm{O}$ sensor de molhamento foi afixado a um suporte de madeira, na altura

TABELA 1 - Esquemas de pulverização empregados nos
diferentes tratamentos para o controle da
requeima da batateira (Solanum tuberosum)

\begin{tabular}{|c|c|}
\hline Tratamento & Esquema de Pulverização \\
\hline 1 - Dimetomorfe + Clorotalonil $^{11}$ & VSD 12 \\
\hline 2 - Dimetomorfe + Clorotalonil $^{11}$ & VSD 14 \\
\hline 3 - Dimetomorfe + Clorotalonil $^{11}$ & VSD 16 \\
\hline $\begin{array}{l}4 \text { - Clorotalonil - Mancozeb - } \\
\text { Oxicloreto Cobre }\end{array}$ & Alternado a cada sete dias \\
\hline 5 - Calendário semanal ${ }^{12}$ & Alternado a cada sete dias \\
\hline 6 - Dimetomorfe + clorotalonil $^{13}$ & VSD 12 \\
\hline 7 - Testemunha & Sem pulverizações \\
\hline $\begin{array}{l}17 \text { Nas semanas em que não foi prevista ne } \\
\text { mancozeb e clorotalonil, alternadament } \\
{ }^{12} \text { Mancozeb-Dimetomorfe-Clorotalonil-c } \\
{ }^{3} \text { Pulverizado somente com fungicida sis } \\
\text { previsão. }\end{array}$ & $\begin{array}{l}\text { uma pulverização, foram aplicados } \\
\text { oxanil; } \\
\text { nico, de acordo com o esquema de }\end{array}$ \\
\hline
\end{tabular}


do terço médio, entre as folhas das plantas em sentido vertical. Os registros foram armazenados em computador para o cálculo dos valores de severidade da doença (VSD).

\section{Modelo de previsão de Wallin (1962) modificado}

O sistema de previsão (modelo de Wallin,1962) foi modificado (Tabela 2), baseado no acúmulo de valores de severidade da doença (VSD), arbitrariamente atribuídos à relação específica entre a duração do período de molhamento foliar e da temperatura média durante o período de molhamento. Após a semeadura dos tubérculos, os VSD's foram calculados diariamente, sendo acumulados até atingir a faixa previamente determinada para cada tratamento (Tabela 1), quando foi realizada a primeira pulverização. As demais pulverizações foram baseadas em novo acúmulo dos valores de severidade. Após a ocorrência de dez dias consecutivos desfavoráveis à doença (VSD's iguais a zero), os VSD's até então acumulados foram desconsiderados, e nova contagem foi iniciada.

O esquema dos tratamentos propostos para estudo neste trabalho difere de outros publicados, pela modificação da tabela de Wallin e pela introdução no tratamento com fungicida sistêmico (dimetomorfe) nos VSD's 12, 14 e 16 de um fungicida protetor (mancozeb ou clorotalonil) em mistura em tanque. Além disto, excetuando-se um tratamento envolvendo o dimetomorfe, os outros tratamentos em que se estudou VSD's de 12 a 16, o tratamento dimetomorfe + mancozeb ou clorotalonil sempre foi alternado com o mancozeb ou clorotalonil na(s) semana(s) em que o sistema de previsão não previra aplicação do fungicida sistêmico em mistura com o protetor (dimetomorfe + mancozeb ou clorotalonil).

\section{Quantificação do progresso da doença e da produção}

Quantificou-se a severidade da requeima aos 57, 64, 71, 78, 85 e 92 dias após a emergência das plantas. Oito plantas por parcela foram previamente marcadas aleatoriamente e utilizadas para as avaliações. Em cada planta avaliou-se a percentagem de área foliar lesionada, com base na escala diagramática desenvolvida por James (1971). A colheita foi realizada em 10/09/98. Os tubérculos

TABELA 2 - Cálculo dos valores de severidade de doença (VSD), em função da duração do período de molhamento foliar e da temperatura média do ar durante o período*

\begin{tabular}{cccccc}
\hline $\begin{array}{c}\text { Temperatura } \\
\text { Média }\left({ }^{\mathbf{0}} \mathbf{C}\right)\end{array}$ & \multicolumn{5}{c}{ Duração do Molhamento Foliar (horas) } \\
\hline $7,2-11,6$ & $0-15$ & $16-18$ & $19-21$ & $22-24$ & $>24$ \\
$11,7-15,0$ & $0-12$ & $13-15$ & $16-18$ & $19-21$ & $>21$ \\
$15,1-26,6$ & $0-9$ & $10-12$ & $13-15$ & $16-18$ & $>18$ \\
\hline $\mathbf{V S D}^{1 \mathbf{1}}$ & $\mathbf{0}$ & $\mathbf{1}$ & $\mathbf{2}$ & $\mathbf{3}$ & $\mathbf{4}$ \\
\hline
\end{tabular}

*Adaptado de Wallin (1962);

${ }^{11}$ Valores de severidade da doença variando de zero a quatro. comercializáveis foram pesados separadamente para cada tratamento. A única doença que surgiu no experimento foi a requeima.

\section{Análise dos dados}

A partir dos dados médios de severidade, foi calculada a área abaixo da curva de progresso da doença (AACPD), utilizando-se o programa AACPD (Torres \& Ventura, 1991) e a taxa de progresso da doença $(r)$ para cada tratamento, ao longo da epidemia. Para o cálculo de $r$, os dados de severidade foram transformados utilizando-se Logit $(Y)=(\operatorname{LN}(Y /(1-Y)))$. As taxas foram então obtidas por meio da regressão linear dos dados transformados em função do tempo. Os valores de AACPD foram padronizados, dividindo-se cada valor pelo número de dias da epidemia. Os valores de AACPD e os dados de produção foram submetidos à análise de variância e ao teste de Tukey, a 5\% de probabilidade. As taxas de progresso foram comparadas calculando-se o intervalo de confiança para a diferença entre as taxas médias de cada dois tratamentos, conforme Campbell e Madden (1990).

\section{RESULTADOS}

$\mathrm{N}$ curvas de progresso da requeima para os diferentes tratamentos ao longo do período de cultivo da batateira, de Maio a Agosto de 1998 (Figura 1). Os tratamentos 1, 2, 3 e 5 proporcionaram um menor progresso da requeima durante todo o ciclo da cultura, nos quais a severidade da doença manteve-se abaixo de 7\%, na última avaliação. O tratamento 4 (aplicação apenas de protetores de acordo com o sistema convencional de controle) apresentou severidade final de 10,7 $\%$. Os tratamentos 6 e 7 (testemunha), embora diferissem quanto à taxa de progresso da requeima (Figura 1A), apresentaram severidade final de $93,7 \%$ e $100 \%$, respectivamente. $\mathrm{O}$ maior desenvolvimento da doença (Figura 1 A e B) em ambos tratamentos foi observado a partir de 50 dias após a emergência (DAE), coincidindo com condições de temperatura e molhamento foliar favoráveis.

Entre os tratamentos baseados no sistema de previsão (1, 2 e 3), o tratamento 1 foi o mais eficiente no controle da requeima, com severidade final de $3,0 \%$, enquanto que o 2 e 3 apresentaram severidade final de 4,5 e 6,8 \%, respectivamente.

Quando se comparou a AACPD, não houve diferença significativa entre os tratamentos 1 a 5 , os quais apresentaram os menores valores (Tabela 3). Entre os tratamentos baseados no sistema de previsão, o tratamento 6 resultou no maior valor de AACPD. O tratamento testemunha apresentou valor de AACPD significativamente superior aos demais.

Entre os tratamentos baseados no sistema de previsão, o tratamento 6 apresentou o maior valor e o 1, o menor valor de $r$, respectivamente. Os tratamentos 2 e 5 não apresentaram diferença significativa quando se comparou o valor de $r$ (Tabelas 4 e 5).

Durante o período de condução do ensaio foram realizadas quatro aplicações com fungicidas sistêmicos e três com protetores no tratamento 5 (Tabela 3 ). O mesmo número 

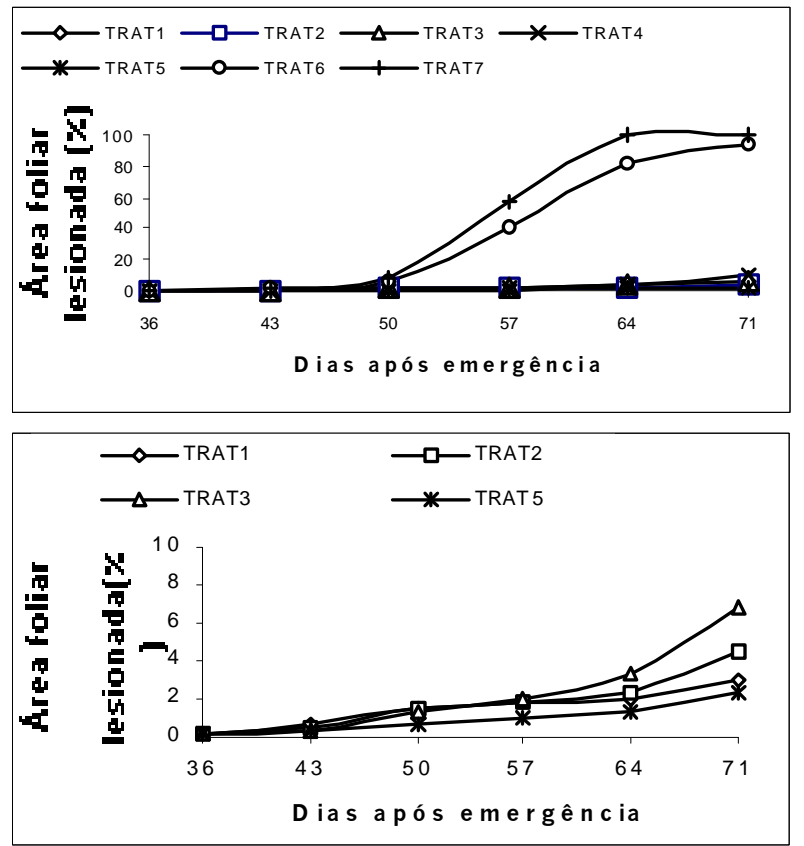

FIG. 1 - Curvas de progresso da requeima da batateira (Solanum tuberosum) para todos os tratamentos (A) e para os tratamentos baseados no esquema de previsão (B). Tratamentos: 1 (VSD 12), 2 (VSD 14) e 3 (VSD 16) empregando-se dimetomorfe + clorotalonil e clorotalonil semanalmente; 4protetores mancozeb, clorotalonil e oxicloreto de cobre alternados semanalmente; 5- calendário fixo: cimoxanil - mancozeb - dimetomorfe clorotalonil; 6 - VSD 12 dimetomorfe + clorotalonil; e 7- testemunha: ausência de controle.

de aplicações de fungicida sistêmico foi realizado no tratamento 1, embora tenha recebido uma aplicação a mais de fungicida protetor. $\mathrm{O}$ menor número de pulverizações foi obtido com o tratamento 3 , sendo três com fungicidas sistêmicos e quatro com protetores. $\mathrm{O}$ tratamento 5 apresentou a maior produtividade, embora estatisticamente não diferisse da produtividade obtida pelos tratamentos 2 e 3 (Tabela 3). Entre os tratamentos baseados no sistema de previsão, o tratamento 6 apresentou a menor produtividade, sendo superior apenas ao tratamento testemunha (sem pulverização).

As condições climáticas prevalecentes durante o período de realização do experimento foram: temperaturas médias em torno de $16,6^{\circ} \mathrm{C}$ com duração média de 7,4 h de molhamento foliar

\section{DISCUSSÃO}

Os resultados obtidos nesse experimento são coerentes com os relatos existentes na literatura, no que diz respeito às condições climáticas que favorecem o desenvolvimento da doença. Segundo Mizubuti \& Fry (1998) epidemias desenvolvendo-se a temperaturas na faixa de $12-16^{\circ} \mathrm{C}$ durante
TABELA 3 - Número de pulverização com fungicidas sistêmicos e protetores, área abaixo da curva de progresso da doença (AACPD) e produtividade entre diferentes tratamentos visando o controle da requeima da batateira (Solanum tuberosum)

\begin{tabular}{|c|c|c|c|c|}
\hline \multirow{2}{*}{ Tratamento $^{1}$} & \multicolumn{2}{|c|}{$\mathbf{N}^{\mathbf{O}}$ Pulverizações } & \multirow{2}{*}{$\begin{array}{l}\text { AACPD } \\
\text { Relativa }\end{array}$} & \multirow{2}{*}{$\begin{array}{c}\text { Rendimento } \\
\text { de tubérculos } \\
\text { (t/ha) }\end{array}$} \\
\hline & Sistêmico & Protetor & & \\
\hline 1 & 4 & 4 & $1,44 \mathrm{a}^{/ 2}$ & $23,6 a b$ \\
\hline 2 & 3 & 4 & $1,61 \mathrm{a}$ & $24,0 \mathrm{ab}$ \\
\hline 3 & 3 & 5 & $2,00 \mathrm{a}$ & $19,4 \mathrm{~b}$ \\
\hline 4 & - & 7 & $2,45 \mathrm{a}$ & $20,1 \mathrm{~b}$ \\
\hline 5 & 4 & 3 & $0,85 \mathrm{a}$ & $26,8 \mathrm{a}$ \\
\hline 6 & 4 & - & $32,51 \mathrm{~b}$ & $12,0 \mathrm{c}$ \\
\hline 7 & - & - & $39,77 \mathrm{c}$ & $8,4 \mathrm{~d}$ \\
\hline $\mathrm{CV} \%$ & - & - & 17,3 & 12,2 \\
\hline
\end{tabular}

Tratamentos: 1) aplicação de dimetomorfe de acordo com VSD (12) + clorotalonil semanalmente; 2) aplicação de dimetormorfe de acordo com VSD (14) + clorotalonil semanalmente; 3 ) aplicação de dimetomorfe de acordo com VSD (16) + clorotalonil semanalmente; 4) aplicação dos protetores mancozeb, clorotalonil e oxicloreto de cobre alternados a cada sete dias; 5) Uso do calendário: mancozeb, dimetomorfe, clorotalonil e cimoxanil, alternados a cada sete dias; 6) aplicação de dimetomorfe + clorotalonil de acordo com VSD (12); e 7) testemunha;

${ }^{12}$ Médias seguidas pela mesma letra não diferem estatisticamente a $5 \%$ de probabilidade pelo teste de Tukey.

TABELA 4 - Taxas médias de progresso da requeima $(r)$ e erros padrões em diferentes tratamentos visando o controle da requeima da batateira (Solanum tuberosum)

\begin{tabular}{ccc}
\hline \hline Tratamento $^{\text {1 }}$ & Taxa (unidades/dia) & Erro padrão \\
\hline 1 & 0,046 & 0,0111 \\
2 & 0,098 & 0,0095 \\
3 & 0,116 & 0,0076 \\
4 & 0,128 & 0,0075 \\
5 & 0,089 & 0,0103 \\
6 & 0,305 & 0,0337 \\
7 & 0,463 & 0,0425 \\
\hline
\end{tabular}

"Tratamentos: 1)aplicação de dimetomorfe de acordo com VSD (12) + clorotalonil semanalmente; 2) aplicação de dimetomorfe de acordo com VSD (14) + clorotalonil semanalmente; 3) aplicação de dimetomorfe de acordo com VSD (16) + clorotalonil semanalmente; 4) aplicação dos protetores mancozeb, clorotalonil e oxicloreto de cobre alternados a cada sete dias; 5) Uso do calendário: mancozeb, dimetomorfe, clorotalonil e cimoxanil, alternados a cada sete dias; 6) aplicação de dimetomorfe + clorotalonil de acordo com VSD (12); e 7) testemunha

a noite, geralmente são mais severas devido ao efeito da temperatura na germinação dos esporângios. Segundo os autores, essa faixa de temperatura favorece a germinação indireta dos esporângios, aumentando seu potencial infetivo e, conseqüentemente, o desenvolvimento da epidemia. A umidade relativa do ar acima de $90 \%$ favorece o desenvolvimento da doença. Períodos de molhamento foliar mais prolongados são necessários à medida que a temperatura distancia-se das condições ótimas para o desenvolvimento de epidemias (Thurston \& Schultz, 1981; Agrios, 1997; 
TABELA 5 - Comparação dos tratamentos através do cálculo dos intervalos de confiança para a diferença das taxas médias de progresso ( $r)$ da requeima da batateira (Solanum tuberosum)

\begin{tabular}{|c|c|c|c|c|c|c|c|}
\hline Tratamento $^{/ 1}$ & 1 & 2 & 3 & 4 & 5 & 6 & 7 \\
\hline 1 & - & & & & & & \\
\hline 2 & $0,046 *$ & - & & & & & \\
\hline 3 & $0,070 *$ & $0,018 \mathrm{~ns}$ & - & & & & \\
\hline 4 & $0,082 *$ & $0,030 *$ & $0,012 \mathrm{~ns}$ & - & & & \\
\hline 5 & $0,043 *$ & $0,009 \mathrm{~ns}$ & $0,027 *$ & $0,039 *$ & - & & \\
\hline 6 & $0,260 *$ & $0,207 *$ & $0,189 *$ & $0,117 *$ & $0,216 *$ & - & \\
\hline 7 & $0,410 *$ & $0,365 *$ & $0,347 *$ & $0,335 *$ & $0,374 *$ & $0,158 *$ & - \\
\hline
\end{tabular}

$\left.{ }^{11} 1\right)$ aplicação de dimetomorfe de acordo com VSD (12) + clorotalonil semanalmente; 2) aplicação de dimetomorfe de acordo com VSD (14) + clorotalonil semanalmente; 3) aplicação de dimetomorfe de acordo com VSD (16) + clorotalonil semanalmente; 4) aplicação dos protetores mancozeb, clorothalonil e oxicloreto de cobre alternados a cada sete dias; 5) Uso do calendário: mancozeb, dimetomorfe, clorotalonil e cimoxanil, alternados a cada sete dias; 6) aplicação de dimetomorfe e clorotalonil de acordo com VSD (12); e 7) testemunha

ns - Diferença não significativa de acordo com intervalo de confiança a $95 \%$ de probabilidade.

* - Diferença significativa de acordo com intervalo de confiança a $95 \%$ de probabilidade .

Zambolim et al., 2000).

No presente trabalho, verificou-se que dependendo das condições climáticas prevalecentes é possível reduzir o número de pulverizações com fungicida sistêmico, sem que haja perda na produção. Os tratamentos baseados nos sistemas de previsão foram eficientes no controle da requeima, proporcionando valores de AACPD e $r$ iguais e, ou inferiores aos obtidos com o uso do sistema convencional de controle. Entre os tratamentos baseados no sistema de previsão, verificou-se uma estreita relação entre o número de aplicações de fungicidas sistêmico e o controle da doença. Os tratamentos que definiram maior número de aplicações de dimetomorfe, mesmo com menor número de pulverização de fungicidas protetores, proporcionaram controle mais eficiente da doença. Porém, o uso apenas de fungicida sistêmico de acordo com o sistema de previsão não foi suficiente para controlar o desenvolvimento da doença (tratamento 6). Os outros tratamentos $(1,2$, e 3$)$ em que o fungicida de contato foi pulverizado em alternância com o sistêmico dimetomorfe, o controle da doença foi mais eficiente. Esse resultado mostra a grande importância da alternância da aplicação do fungicida protetor, sempre que o sistema de previsão, não prever nenhuma aplicação do fungicida sistêmico, sob condições altamente favoráveis a requeima. Este esquema além de ser eficiente, previne o surgimento de resistência na população de $P$. infestans. O fungicida sistêmico aplicado sem a alternância do fungicida de contato, proporciona controle incompleto da requeima.

O tratamento 1 igualou ao calendário semanal (tratamento 5), em relação ao número de pulverizações com dimetomorfe, no controle da requeima.

O uso do sistema de previsão reduziu o número de pulverizações (tratamentos 2 e 3 ), e também permitiu uma melhor distribuição da aplicação dos fungicidas ao longo do período de cultivo. Resultados semelhantes foram obtidos por Nazareno et al. (1999), utilizando o modelo de previsão de Wallin (1962) para o controle da requeima. Segundo os autores, o número de aplicações foi reduzido em $16 \%$ na safra das águas e 33\% na safra das secas. Michel et al. (1997a, 1997b), utilizando o mesmo modelo nos intervalos de VSD 13 - 15, 18 a 20 e 23 - 25, obtiveram controle equivalente ao observado no sistema convencional de aplicação de fungicidas, com redução superior a 50\% do número de aplicações.

No modelo de previsão de Wallin (1962) a previsão da requeima é feita com base na umidade relativa do ar $(\geq$ $90 \%$ ) e temperatura média diária. Neste trabalho, o modelo foi modificado para duração do período de molhamento foliar e temperatura média durante esse período. Porém, foram mantidos os mesmos períodos de duração, originalmente designados para umidade relativa. Segundo Jones (1986) a umidade relativa não é uma variável adequada para se prever o molhamento foliar, podendo resultar em previsões incorretas. Há uma tendência à formação inicial de orvalho, com umidade relativa mais baixa no inverno do que no verão, e a perda de energia pela folha depende da umidade absoluta do ar e da formação de nuvens. No presente trabalho foram observados períodos de molhamento foliar com a umidade relativa do ar em torno de $80 \%$. Portanto, o uso do molhamento foliar nos intervalos originalmente definidos para umidade relativa no modelo pode ter superestimado o desenvolvimento da doença, resultando em maior número de pulverizações. Além disso, a determinação do molhamento foliar por meio de sensores está sujeita a erros. As características físicas e químicas dos sensores são completamente diferentes das de uma folha, afetando a precisão da previsão da doença (Campbell \& Madden, 1990).

O calendário de pulverização semanal utilizado neste trabalho foi bastante moderado e criterioso. Normalmente, os produtores (principalmente aqueles que utilizam a cultivar Bintje) realizam duas a três pulverizações por semana, chegando a pulverizar diariamente quando as condições climáticas são favoráveis à requeima.

Apesar de se ter utilizado a cultivar Bintje, altamente suscetível à requeima, o sistema de previsão apresentou eficiência comparável ao calendário semanal. Mas, torna-se imprescindível a aplicação de fungicida protetor na semana 


\section{R.V. Costa et al.}

em que o sistema de previsão não preveja aplicação de fungicida sistêmico, para viabilizar o sistema de previsão de Wallin (1962), empregando-se como fungicida sistêmico o dimetomorfe.

Entretanto, os valores de VSD devem ser ajustados para cada cultivar e condições climáticas locais, para seu uso em diferentes localidades. Para as condições climáticas de Viçosa - MG, os VSD's 12 e 14 apresentaram maior eficiência em prever a ocorrência de epidemias de requeima.

Segundo os resultados das análises empregadas, a taxa de progresso da doença $(r)$ discriminou melhor os tratamentos do que a AACPD, embora a diferença das taxas de progresso entre alguns tratamentos tenha sido muito baixa, em termos biológicos. Curvas de progresso com um mesmo valor de AACPD podem apresentar diferenças quanto ao tempo inicial, à severidade inicial e final e à taxa de progresso da doença. Resultados semelhantes foram obtidos por Nazareno et al. (1999), que alertam quanto à necessidade de atenção quando se usa apenas a AACPD em trabalhos dessa natureza.

Em função da alta intensidade e velocidade de progresso da doença, observou-se de um modo geral, entre os tratamentos, uma relação negativa entre a intensidade de doença e a produção. $\mathrm{O}$ tratamento 2 baseado no sistema de previsão permitiu reduzir o número de aplicações com fungicida sistêmico, sem ocasionar perda significativa na produção.

\section{REFERÊNCIAS BIBLIOGRÁFICAS}

AGRIOS, G.N. Plant Pathology. $4^{\mathrm{a}}$ edição, editora Academic Press. 1997.

BERGAMIN FILHO, A. \& AMORIM, L. Sistemas de previsão e avisos. In: Bergamin Filho, A., Kimati, H. \& Amorim, L. (Eds.) Manual de Fitopatologia: princípios e conceitos. São Paulo: Agronômica Ceres, 1995. pp. 602-626.

CAMPBELL, C.L. \& MADDEN, L.V. Introduction to plant disease epidemiology. New York, John Wiley \& Sons. 1990.

CHALFOUN, S.M. \& LIMA, R.D. de. Influência do clima sobre a incidência de doenças infecciosas. Informe agropecuário 12:3136. 1986.

HOOKER, W.J. Compendium of potato diseases. St. Paul: APS Press, 1981.

HYRE, R.A. Progress in forecasting late blight of potato and tomato. Plant Disease Reporter 38:245-253. 1954.

JAMES, W.C. An illustrated series of assessment keys for plant disease, their preparation and usage. Canadian Plant Disease Survey 51:39-65. 1971.

JONES, A.L. Role of wet periods in predicting foliar disease. In: Leonard, K.J. \& Fry, W.E. (Eds.). Plant disease epidemiology: population dynamics and management. New York: MacMillan Publishing Company, 1986. pp. 87-89.

LOPES, C.A. \& SANTOS, J.R.M. Doenças do tomateiro. Brasília: EMBRAPA/CNPH, 1994.

LORENA, B.A. cultura da batata. Boletim de Agricultura 14:634640. 1913.

MICHEL, C.A., MENDES, C.S. \& REIS, E.M. Validação de sistemas de previsão de epidemias de Phytophthora infestans na cultura da batata. I - safra 1996/1997. Fitopatologia Brasileira 22:285. 1997a.

MICHEL, C.A., MENDES, C.S. \& REIS, E.M. Validação de sistemas de previsão de epidemias de Phytophthora infestans na cultura da batata. II - safrinha 1997. Fitopatologia Brasileira 22:285. $1997 b$.

MIZUBUTI, E.S.G. \& FRY, W. Temperature effects on developmental stages of isolates from three clonal lineages of Phytophthora infestans. Phytopathology 88:837-843. 1998.

NAZARENO, N.R.X., SCOTTI, C.A., MAFIOLETTI, R.L. \& BOSCHETTO, N. Controle da requeima da batata através do monitoramento das variáveis climáticas. Fitopatologia Brasileira 24:170-174. 1999.

PITBLADO, R.E. Development of a weather-timed fungicide spray program for field tomatoes. Canadian Journal of Plant Pathology 10:371. 1988. (Abstract).

ROYLE, D.J. \& SHAW, M.W. The costs and benefits of disease forecasting in farming practice. In: Clifford, B.C. \& Lester, E. (Eds.). Control of plant disease: costs and benefits. Blackwell, Oxford. 1988, pp. 231-246.

STEVENSON, W.R. An integrated program for managing potato late blight. Plant Disease 67:1047-1048. 1983.

TORRES, J.C. \& VENTURA, J.A. AACPD: um programa para calcular a área e o volume abaixo da curva de progresso da doença. Fitopatologia Brasileira 16:52. 1991. (Resumo).

THURSTON, H.D. \& SCHULTZ, O. Late blight. In: Hooker, W.J. (Ed.). Compendium of potato disease. St. Paul. APS PRESS, 1981, pp. 40-42,

VALE, F.X.R., ZAMBOLIM, L., CHAVES, G.M. \& CORREIA, L.G. Avaliação fitossanitária da cultura do tomateiro em regiões produtoras de Minas Gerais e Espírito Santo. Fitopatologia Brasileira 17:211. 1992 (Resumo).

WALLIN, J.R. Summary of recent progress in predicting late blight epidemics in United States and Canada. American Potato Journal 39:306-312. 1962.

ZAMBOLIM, L., VALE, F.X.R., CRUZ FILHO, J. \& CHAVES, G.M. Emprego da Calda Viçosa na cultura do tomateiro ( $L$. esculentum) para o controle de doenças da parte aérea. Viçosa: UFV, Informe técnico, 66, 1990.

ZAMBOLIM, L., VALE, F.X.R. \& COSTA, H. Doenças causadas por fungos em batata. In: Zambolim, L., Vale, F.X.R. \& Costa, H. (Eds). Controle de doenças de plantas. Hortaliças-Vol. 1. Visconde do Rio Branco, Suprema Gráfica e Editora, 2000, p.173-207. 